\title{
Self-Reported Adherence after Overnight Switching from Immediate- to Extended-Release Pramipexole in Parkinson's Disease
}

\author{
Mutsumi Iijima*, Mikio Osawa, Kenji Maruyama, Shinichiro Uchiyama, Kazuo Kitagawa \\ Department of Neurology, Tokyo Women's Medical University School of Medicine, Tokyo, Japan \\ Email: ${ }^{*}$ mutumi@nij.twmu.ac.jp
}

Received 18 January 2015; accepted 1 February 2015; published 5 February 2015

Copyright (C) 2015 by authors and Scientific Research Publishing Inc.

This work is licensed under the Creative Commons Attribution International License (CC BY).

http://creativecommons.org/licenses/by/4.0/

(c) (i) Open Access

\begin{abstract}
Background: Drug adherence decreased in patients with Parkinson's disease (PD) because of taking many different types of drugs. We evaluated drug adherence after switching from immediate-release (IR) to once-daily extended-release (ER) pramipexole (PPX) in PD. Methods: This study included 35 PD patients (20 men, 15 women); 10 were taking oral PPX alone, and 25 were also using another anti-PD drug. PPX-IR was switched overnight to PPX-ER without gradual tapering. One month after switching, improvement in timing adherence and reduction in medication burden were evaluated by a questionnaire using a visual analog scale (VAS) (0: No change; 10: Better). Motor function was assessed using part III of the Unified Parkinson's Disease Rating Scale (UPDRS). Results: The VAS score for improvement in timing adherence was $8.1 \pm 0.5$ (mean \pm standard error), and that for reduction in medication burden was $7.3 \pm 0.6$. There was a significant negative correlation $(\rho=-0.43, p=0.01)$ between the VAS score and number of types of medications. The UPDRS part III score improved significantly after switching $(p<0.01)$. Only one patient discontinued PPX-ER after switching. Conclusion: Switching from PPX-IR to once-daily PPX-ER is safe and improves motor function in patients by improving timing adherence and reducing medication burden.
\end{abstract}

\section{Keywords}

Parkinson's Disease, Timing Adherence, Pramipexole, Extended-Release, Immediate-Release, Motor Function

\footnotetext{
*Corresponding author.
}

How to cite this paper: lijima, M., Osawa, M., Maruyama, K., Uchiyama, S. and Kitagawa, K. (2015) Self-Reported Adherence after Overnight Switching from Immediate- to Extended-Release Pramipexole in Parkinson's Disease. Advances in Parkinson's Disease, 4, 13-19. http://dx.doi.org/10.4236/apd.2015.41003 


\section{Introduction}

Maintaining good drug adherence requires a feasible treatment plan that a patient can follow and continue that regimen between each patient and their health care provider. Patients with Parkinson's disease (PD) take many different drugs even in the early stage, and as PD progresses, the doses and types of drugs increase, and the times for taking these medications become more complex. Research on drug adherence in PD has reported "not taking drugs on time”, in other words, decreased timing adherence, as an issue [1] [2]. Poor adherence in PD is directly correlated with the complexity of drug regimen, with the depression, and with cognitive impairment [2]-[5]. PD patients prefer drugs that can be taken less often each day and in lower amounts [6] [7]. Moreover, better adherence with anti-PD drugs taken once daily, compared to drugs taken multiple times daily, has been shown [2] [8].

Once-daily extended-release formulations of non-ergot dopamine agonists (DAs) as anti-PD drugs are now available. However, studies on adherence when switching from multiple-daily to once-daily administration of these drugs have not been conducted. Pamipexole is a non-ergot dopamine agonist, and directly stimulates as a partial/full agonist at the D2, D3 and D4 receptors in the striatum. In this study, we investigated the effects on adherence of switching from the pramipexole immediate-release (PPX-IR) to pramipexole extended-release (PPX-ER) formulation.

\section{Methods}

\subsection{Subjects}

We conducted a hospital-based study involving 35 consecutive patients with PD in the Department of Neurology at the Tokyo Women's Medical University Hospital between July 2012 and November 2013. Subjects were included 35 PD patients (20 men, 15 women) ranging in age from 49 to 83 years (mean \pm SD: $67.8 \pm 9.1$ years), with disease duration of 7 to 132 months (65.1 \pm 31.1 months). PD severity was Hoehn and Yahr stage I in 5, II in 19, III in 10, and IV in 1 patient. Cognitive function was evaluated using the Mini-Mental State Examination (MMSE), and MMSE scores for the patients with PD ranged from 21 to 30 points (21 points in 1 patient, and another patients were above 25 points). The oral PPX dose before switching was $1.83 \pm 0.5 \mathrm{mg}$, including 10 patients taking the PPX alone and 25 patients also taking other anti-PD drugs. These other drugs included levodopa in 22 (mean: $288 \pm 120 \mathrm{mg}$ ), zonisamide in 11 (40.1 $\pm 23.1 \mathrm{mg}$ ), selegiline in 9 (5.3 $\pm 2.0 \mathrm{mg})$, amantadine in 6 (166 $\pm 40.8 \mathrm{mg})$, trihexyphenidyl in $5(2.4 \pm 0.9 \mathrm{mg})$, and entacapone in $2(400.0 \pm 141.4 \mathrm{mg})$ patients. The total number of drug types, including anti-PD drugs and other drugs, was 1 drug in 7, 2 drugs in 7, 3 drugs in 7, 4 drugs in 9, 5 drugs in 1, 6 drugs in 1, 7 drugs in 1, and 9 drugs in 2 patient. This study was performed in accordance with the guidelines of the Committee of Medical Ethics of Tokyo Women's Medical University. Informed consent was obtained from patients before study participation.

\subsection{Switching from PPX-IR to PPX-ER}

PPX-IR was switched overnight to PPX-ER without any gradual tapering. The dosage of the ER formulation was the same as the IR formulation in 27 patients; in 8 patients without a dosage corresponding to the IR formulation, the dose was increased (0.25-mg increase, 5 patients; 0.5 -mg increase, 3 patients). The daily dose of PPX was $1.83 \pm 0.5 \mathrm{mg}$ before switching and $1.90 \pm 0.6 \mathrm{mg}$ after switching. The switched dose was significantly higher $(\mathrm{p}<0.01)$.

\subsection{Evaluation of Drug Adherence and Medication Burden}

Patients were asked the following questions 4 - 6 weeks after switching to PPX-ER: Q1. Have you forgotten to take your anti-PD drug? Q2. Do you now take your medication on time with once-daily dosing? (timing adherence) and Q3. Has the burden of taking your medication decreased with once-daily dosing? Responses for Q 2 and Q3 were assessed using a visual analog scale (VAS): (0: No change; 10: Better) (Figure 1).

\subsection{Patient Survey about Motor Function and Mood}

Patients filled out a survey form with the following questions: Q1. How are your daily activities compared to previously? Q2. How are your evening activities compared to previously? Q3. How are your morning activities 


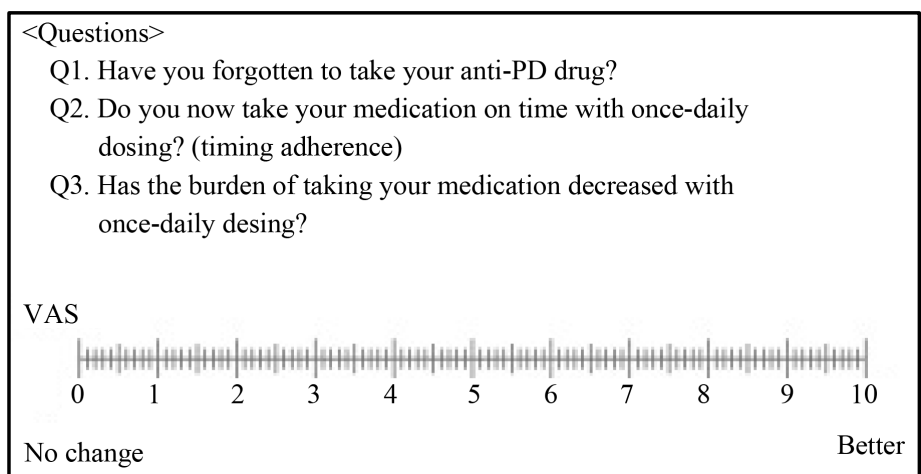

Figure 1. Questionnaire of drug adherence and medication burden Responses for Q2 and Q3 were assessed using a visual analog scale (VAS) (0: No change; 10: Better).

compared to previously? and Q4. How is your mood compared to previously? There were 7 grades of response (much worse, worse, somewhat worse, no change, somewhat better, better, and much better) (Figure 2).

\subsection{Motor Function}

Motor function was assessed before and 4 - 6 weeks after switching using part III of the Unified Parkinson's Disease Rating Scale (UPDRS) that is composed of 14 items (speech, facial expression, tremor at rest, action or postural tremor of hands, rigidity, finger taps, hand movements, rapid alternating movements of hands, leg agility, arising from chair, posture, gait, postural stability and body bradykinesia and hypokinesia).

\subsection{Statistical Analysis}

Spearman's rank correlation was used to examine the correlation between the VAS scores for questions 2 and 3 about taking medications with each of the following: number of types of medications, age, severity, disease duration, and MMSE score. One-way ANOVA was used to compare the single-drug and multiple-drug groups. The paired $t$-test was used to compare doses and analyze UPDRS changes before and after switching. The level of significance was $\mathrm{p}<0.05$.

\section{Results}

\subsection{Adherence}

The responses to Q1 (Have you forgotten to take your PPX-ER?) were 100 \% “no” and 0 \% “yes”.

The VAS score for Q2 (Do you now take your medication on time with once-daily dosing?) was 10 in $48 \%$ of patients and $\geq 8$ in $74 \%$ of patients (Figure 3, left). The VAS score (mean \pm SE) for all patients was $8.1 \pm 0.5$, including $9.4 \pm 0.8$ in the single drug group and $7.6 \pm 0.5$ in the multiple drug group. The single-drug group took their medication more on time, but the difference was not significant ( $p=0.07)$. Two patient using 6 and 9 concomitant drugs responded "no change".

There was a significant negative correlation between the VAS score and the number of types of medications $(\rho=-0.43, \mathrm{p}=0.01)$. There was no significant correlation between the VAS score and age, PD severity, disease duration, or MMSE score.

\subsection{Medication Burden}

The VAS score for Q3 (Has the burden of taking your medication decreased with once-daily dosing?) was 10 in $49 \%$ of patients and $\geq 8$ in $61 \%$ of patients (Figure 3 , right).

The VAS score (mean $\pm \mathrm{SE}$ ) for all patients was $7.3 \pm 0.6$, including $8.6 \pm 1.1$ in the single-drug group and $6.9 \pm 0.7$ in the multiple-drug group. The single-drug group reported more of a reduction in medication burden, but the difference was not significant $(\mathrm{p}=0.18)$. Four patients using concomitant drugs in the multiple drug group responded "no reduction in burden". 


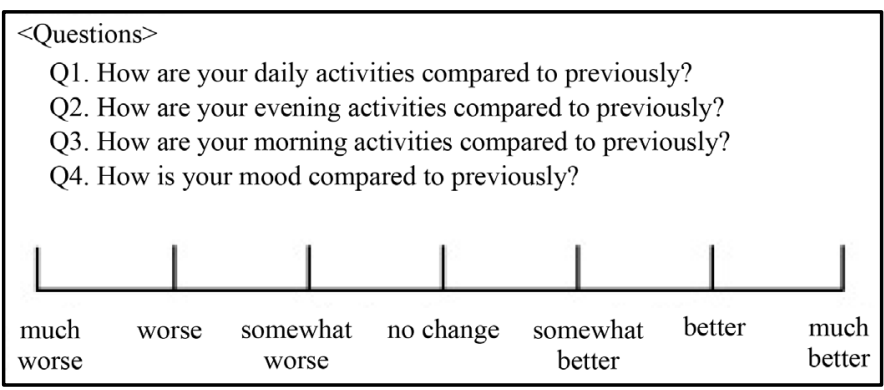

Figure 2. Patient survey about motor function and mood. Patients filled out a survey form with the questions.

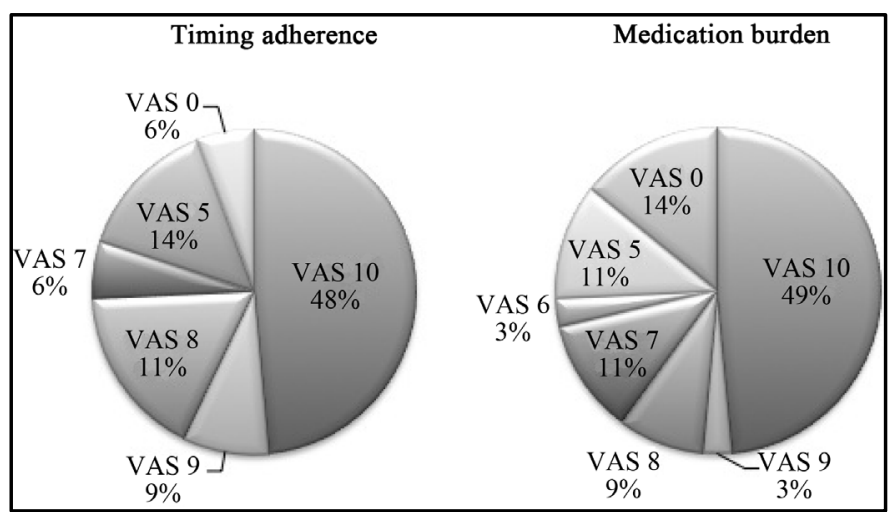

Figure 3. Survey results for timing adherence (left) and medication burden (right) after switching. VAS: visual analog scale; 0: No change; 10: Better.

There was no significant correlation between the VAS score for reduction in medication burden and age, PD severity, disease duration, MMSE score, or number of types of medications.

\subsection{Patient Survey about Motor Function and Mood (Figure 4)}

1) Daily activities

The responses in 27 patients switched to the same dose were: no change $59 \%$, better $19 \%$, somewhat better $11 \%$, somewhat worse $7 \%$, and worse $4 \%$. On the other hand, the responses in the 8 patients with an increased dose were: no change $50 \%$, much better $12 \%$, better $13 \%$, and somewhat better $25 \%$; none reported subjective worsening.

2) Evening activities

The responses in the patients switched to the same dose were: no change $74 \%$, better $4 \%$, somewhat better $15 \%$, and somewhat worse $7 \%$. The responses in patients with an increased dose were: no change $75 \%$, much better $12 \%$, and better $13 \%$, with none reporting subjective worsening.

3) Morning activities

The responses in the patients switched to the same dose were: no change $74 \%$, somewhat better $19 \%$, and somewhat worse $7 \%$. The responses in patients with an increased dose were: no change $87 \%$ and somewhat better $13 \%$, with none reporting subjective worsening.

4) Mood

The responses in the patients switched to the same dose were: no change $55 \%$, much better $4 \%$, somewhat better $22 \%$, somewhat worse $11 \%$, worse $4 \%$, and much worse $4 \%$. The responses in patients with an increased dose were: no change $50 \%$, better $12 \%$, and somewhat better $38 \%$, with none reporting subjective worsening.

\subsection{UPDRS Part III}

In the group switched to the same dose, the UPDRS part III score ranged from 3 to 28 (10.4 \pm 1.3$)$ before 


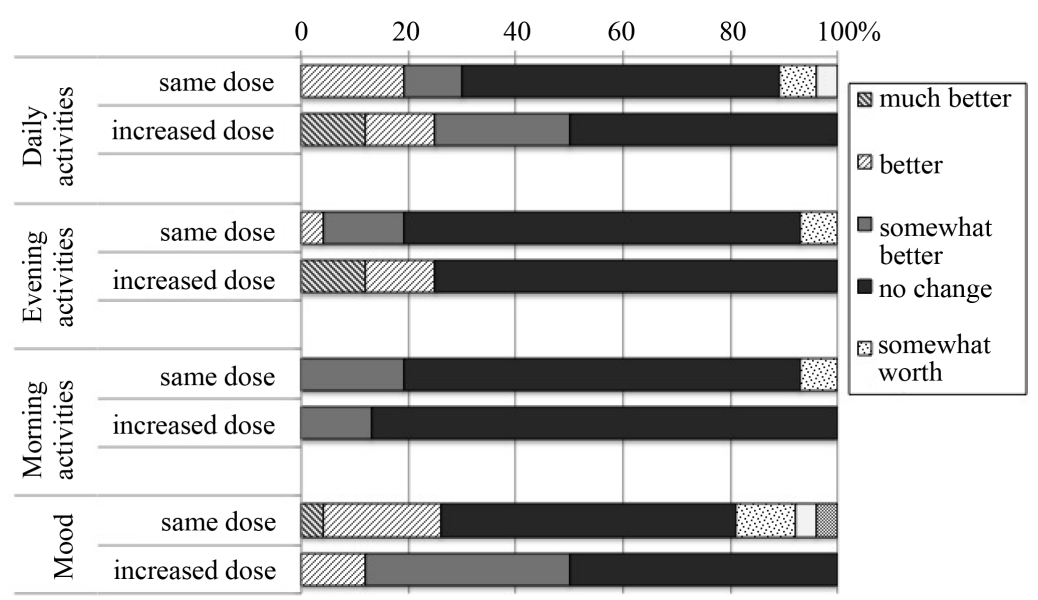

Figure 4. Patient survey about motor function and mood in the group switched to the same dose and an increased dose.

switching and 3 to $26(8.8 \pm 1.0)$ after switching. In the group switched to an increased dose, the scores ranged from 2 to $22(11.3 \pm 4.1)$ before switching and from 1 to $22(7.3 \pm 2.3)$ after switching. After switching to the extended release formulation, the UPDRS part III scores improved significantly in both groups $(\mathrm{p}<0.01)$.

\subsection{Safety}

Because of chest discomfort and worsening mood, one patient discontinued PPX-ER and was switched back to PPX-IR. In addition, there was mild somnolence in 5 , constipation in 1 , and mild worsening of visual hallucinations in 1 patient.

\section{Discussion}

\subsection{Adherence by Switching to PPX-ER}

Adherence includes the percentage of doses taken vs. the total number of doses prescribed (total adherence), the percentage of days a drug is actually taken as prescribed vs. the total number of days prescribed (days adherence), and the percentage of taking a drug at a correct time vs. the prescribed frequency of administration (timing adherence). In a multicenter collaborative study of drug adherence in PD, total adherence, days adherence, and timing adherence were significantly better in a group taking a DA once-daily compared to 3 times daily [2] [8]. In particular, timing adherence differed markedly between groups: $\geq 90 \%$ in the once-daily group, but it decreased to about $25 \%$ in the 3-times-daily group. Even the impact of twice-daily dosing was a marked decrease in adherence to $33 \%$ [2] [8]. Based on the above reports, the present study focused on and evaluated timing adherence using a VAS patient survey. After patients switched from multiple daily PPX-IR to once-daily PPX-ER, timing adherence improved, and medication burden was reduced. In addition, timing adherence was negatively correlated with the number of types of medications being taken. In patients using $\geq 6$ types of medications, timing adherence did not improve even after switching to PPX-ER. Drug non-adherence reportedly increases 1.86 times with a 100-mg increase in levodopa dose, and there is a negative correlation with total number of drugs, with a 1.68-fold increase in non-adherence with each additional drug [5] [9]. Factors associated with decreased adherence besides multiple drugs and complicated dosing regimens include mood disturbance, decreased cognitive function, poor control, long disease duration, educational level, absence of a spouse, younger age, and low income [2]-[5]. Attention to these patient factors and therapeutic interventions are essential to maintain good adherence.

A limitation of this study is that we evaluated adherence by using the self-reported questionnaires. In previous studies on adherence, differences in adherence rates have been observed between evaluations using patient surveys and evaluations using electronic monitoring systems of the actual number of doses taken and times when taken, with a trend toward more over-estimation on patient surveys [1] [2] [10]-[12]. In this study, although almost patients showed normal MMSE scores, and none of the patients responded that they "forgot to take PPX- 
ER”, because of evaluation of timing adherence using a VAS patient survey, some discrepancy between prescribed times and actual times taken may have arisen. However, in every clinical practice, it was useful to have a simple questionnaire to assess the adherence to therapy in PD.

\subsection{Changes in Motor Function by Switching to PPX-ER}

In some patients switched to the same dose, there was worsening of daily, evening, and morning activities and of mood. In patients switched to an increased dose, there was no worsening of these parameters. However, the UPDRS part III scores increased significantly ( $p<0.01$ ) not only in the increased dose group, but also in patients switched to the same dose. Overnight switching from PPX-IR to PPX-ER can be safely accomplished without worsening of symptoms in about 85\% of patients [7] [13]-[15]. Moreover, improvement in UPDRS part III scores and reduction in off-times in advanced PD patients have been reported [7] [14]. Because of some issues with corresponding dosage formulations in this study, the mean dose increased after switching, and this may have been a factor in the improved UPDRS part III scores. There was also significant improvement, however, in patients switched to the same dose, and taking into consideration the effect of drug adherence on therapeutic efficacy and QOL [16], the on-time dosing with better drug adherence and longer lasting drug delivery with the extended release formulation probably contributed to improved motor function.

\section{Conclusion}

This study showed that switching from PPX-IR to once-daily PPX-ER was safe and improved motor function in patients by improving timing adherence and reducing medication burden.

\section{Disclosures of Conflicts of Interest}

None of the authors report any disclosures.

\section{References}

[1] Leopold, N.A., Polansky, M. and Hurka, M.R. (2004) Drug Adherence in Parkinson's Disease. Movement Disorders, 19, 513-517. http://dx.doi.org/10.1002/mds.20041

[2] Grosset, D.G., Antonini, A., Canesi, M., Pezzoli, G., Lees, A., Shaw, K., Esther Cubo, E., Martinez-Martin, P., Rascol, O., Negre-Pages, L., Senard, A., Schwarz, J., Strecker, K., Reichmann, H., Storch, A., Löhle, M., Stocchi, F. and Grosset, K. (2009) Adherence to Antiparkinson Medication in a Multicenter European Study. Movement Disorders, 24, 826-832. http://dx.doi.org/10.1002/mds.22112

[3] Kulkarni, A.S., Balkrishnan, R., Anderson, R.T., Edin, H.M., Kirsch, J. and Stacy, M.A. (2009) Medication Adherence and Associated Outcomes in Medicare Health Maintenance Organization-Enrolled Older Adults with Parkinson's Disease. Movement Disorders, 23, 359-365. http://dx.doi.org/10.1002/mds.21831

[4] Valldeoriola, F., Coronell, C., Pont, C., Cámara, C., Gaig , C. and Compta, Y., on Behalf of the Members of the ADHESON Study Group (2011) Socio-Demographic and Clinical Factors Influencing the Adherence to Treatment in Parkinson's Disease: The ADHESON Study. European Journal of Neurology, 13, 980-987. http://dx.doi.org/10.1111/j.1468-1331.2010.03320.x

[5] Daley, D.J., Myint, P.K., Gray, R.J. and Deane, K.H. (2012) Systematic Review on Factors Associated with Medication Non-Adherence in Parkinson's Disease. Parkinsonism \& Related Disorders 18, 1053-1061. http://dx.doi.org/10.1016/j.parkreldis.2012.09.004

[6] Schapira, A.H.V., Barone, P., Hauser, R.A., et al. (2013) Patient-Reported Convenience of Once-Daily versus ThreeTimes-Daily Dosing during Long-Term Studies of Pramipexole in Early and Advanced Parkinson' s Disease. European Journal of Neurology, 20, 50-56. http://dx.doi.org/10.1111/j.1468-1331.2012.03712.x

[7] Takanashi, M., Shimo, Y., Hatano, T., Oyama, G. and Hattori, N. (2013) Efficacy and Safety of a Once-Daily Extended-Release Formulation of Pramipexole Switched from an Immediate-Release Formulation in Patients with Advanced Parkinson's Disease: Results from an Open-Label Study. Drug Research, 63, 639-643. http://dx.doi.org/10.1055/s-0033-1351257

[8] Grosset, K.A. and Grosset, D.G. (2007) Effect of Educational Intervention on Medication Timing in Parkinson’s Disease: A Randomized Controlled Trial. BMC Neurology, 7, 20. http://dx.doi.org/10.1186/1471-2377-7-20

[9] Santos-García, D., Prieto-Formoso, M. and Fuente-Fernández, R. (2012) Levodopa Dosage Determines Adherence to Long-Acting Dopamine Agonists in Parkinson’s Disease. Journal of the Neurological Sciences, 318, 90-93. 
http://dx.doi.org/10.1016/j.jns.2012.03.018

[10] Grosset, K.A., Bone, I., Reid, J.L. and Grosset, D.G. (2006) Measuring Therapy Adherence in Parkinson’s Disease: A Comparison of Methods. Journal of Neurology, Neurosurgery \& Psychiatry, 77, 249-251. http://dx.doi.org/10.1136/jnnp.2005.064709

[11] Elm, J.J., Kamp, C., Tilley, B.C., Guimaraes, P., Fraser, D., Deppen, P., Brocht, A., Weaver, C., Bennett, S., for NINDS NET-PD Investigators and Coordinators (2007) Self-Reported Adherence versus Pill Count in Parkinson's Disease: The NET-PD Experience. Movement Disorders, 22, 822-827. http://dx.doi.org/10.1002/mds.21409

[12] Tarrants, M.L., Denarié, M.F., Castelli-Haley, J., Millard, J. and Zhang, D. (2010) Drug Therapies for Parkinson’s Disease: A Database Analysis of Patient Compliance and Persistence. The American Journal of Geriatric Pharmacotherapy, 8, 374-383. http://dx.doi.org/10.1016/j.amjopharm.2010.08.001

[13] Rascol, O., Barone, P., Hauser, R.A., Mizuno, Y., Poewe, W., Schapira, A.H.V., Salin, L., Sohr, M. and Debieuvre, C. (2010) Efficacy, Safety, and Tolerability of Overnight Switching from Immediate- to Once Daily Extended-Release Pramipexole in Early Parkinson’s Disease. Movement Disorders, 25, 2326-2332. http://dx.doi.org/10.1002/mds.23262

[14] Mizuo, Y., Yamamoto, M., Kuno, S., Hasegawa, K., Hattori, N., Kagimura, T., Sarashina, A., Rascol, O., Schapira, A.H.V., Barone, P., Hauser, R., Poewe, W., The Pramipexole ER Study Group (2012) Efficacy and Safety of Extended- versus Immediate- Release Pramipexole in Japanese Patients with Advanced and L-dopa-Undertreated Parkinson Disease: A Double-Blind, Randomized Trial. Clinical Neuropharmacology, 35, 174-181. http://dx.doi.org/10.1097/WNF.0b013e31825f77b9

[15] Schapira, A.H., Barone, P., Hauser, R.A., Mizuno, Y., Rascol, O., Busse, M., Debieuvre, C., Fraessdorf, N. and Poewe, W. (2013) Success Rate, Efficacy, and Safety/Tolerability of Overnight Switching from Immediate- to Extended-Release Pramipexole in Advanced Parkinson's Disease. European Journal of Neurology, 20, 180-187. http://dx.doi.org/10.1111/j.1468-1331.2012.03822.x

[16] Bainbridge, J.L. and Ruscin, J. (2009) Challenges of Treatment Adherence in Older Patients with Parkinson's Disease. Drugs \& Aging, 26,145-155. http://dx.doi.org/10.2165/0002512-200926020-00006 
Scientific Research Publishing (SCIRP) is one of the largest Open Access journal publishers. It is currently publishing more than 200 open access, online, peer-reviewed journals covering a wide range of academic disciplines. SCIRP serves the worldwide academic communities and contributes to the progress and application of science with its publication.

Other selected journals from SCIRP are listed as below. Submit your manuscript to us via either submit@scirp.org or Online Submission Portal.
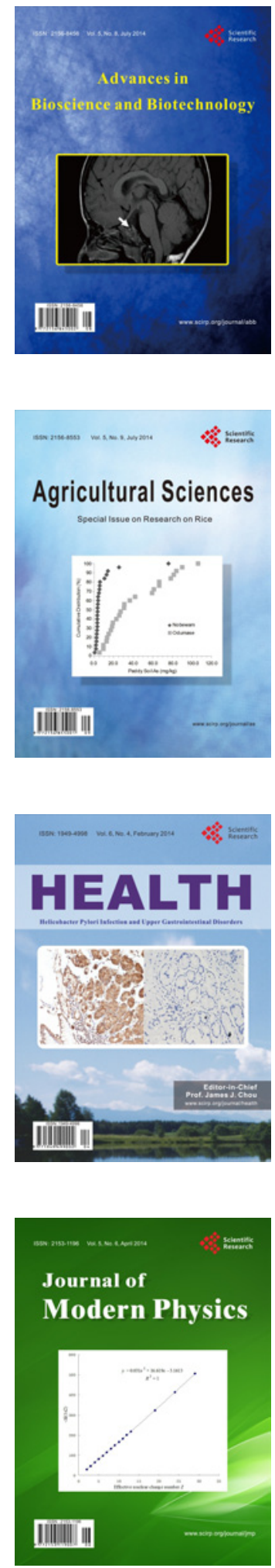
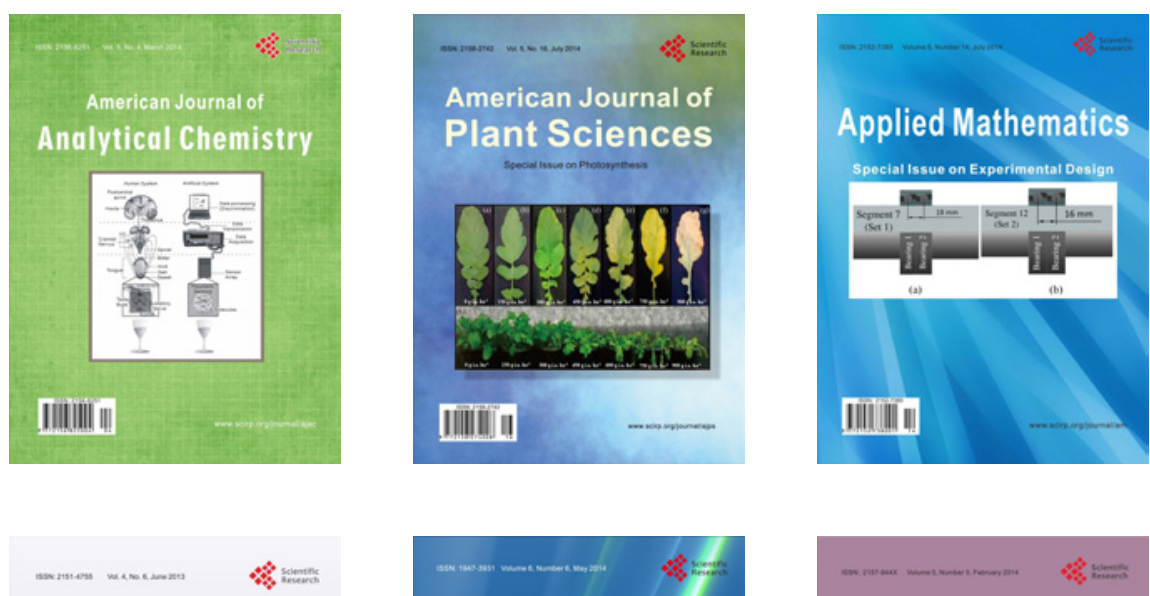

Creative Education
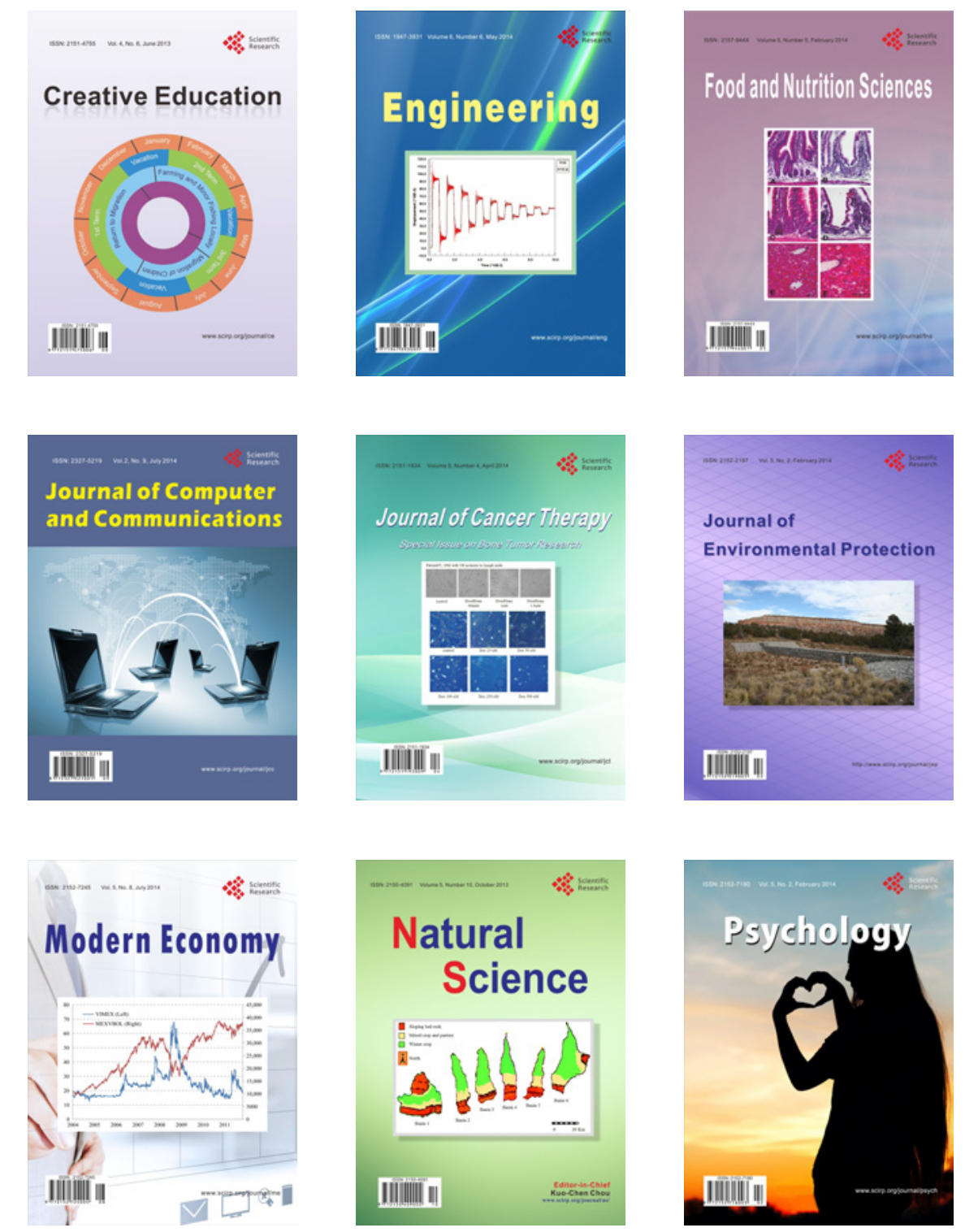